exploration in order to discover the obstruction. I therefore dissected the skin back, and amputated the hind extremities at the hip joints. I then discovered the nature of the foetus and gave up all hope of being able to effect delivery without sacrificing the heifer. When the latter was killed I extracted the calf, a photograph of which is reproduced in Plate IV. It was fully developed, and it had given signs of being alive until some time after labour had commenced. It had two well-developed heads and spines, a third rudimentary spine (three tails), four fore-legs, and two hind-legs.

\title{
A CASE OF OPEN JOINT SUCCESSFULLY TREATED.
}

By Joseph Abson, F.R.C.V.S., Sheffield.

UPON reading the article by Professor $F$. Smith in your September number, on "Some Joint Diseases of the Horse," I am constrained to record a case that $I$ attended in the months of July and August last, and which, I think, may be of interest to your readers.

The subject, a three-years-old Hackney filly, was left standing at a door attached to a two-wheeled vehicle, and without any one in charge, with the result that she bolted and ultimately came to grief by overturning the trap and smashing it up, but at the time it was supposed that she had escaped injury herself. She was taken home and straightway turned out into a meadow adjoining the house of the owner. On the following morning the mare was noticed to be lame on the off hind leg. She was brought home, and the limb was well washed, and it was then noticed that she had a small wound on the hock. I was sent for, and on examination discovered a perfectly round opening into the true hock joint, quite the size of a threepenny piece. This was as clearly defined as though cut by a saddler's punch, was on the inner aspect, well to the front of the joint, and nearly at the bottom of the capsule. The white glistening articular cartilage of the astragalus was plainly visible, even at a distance of several feet away, and any movement of the joint could readily be seen.

Little synovia appeared to have escaped, from what I could learn, and the lameness for such a serious lesion was only trifling. My prognosis was most unfavourable. The mare was put into a loose box where she was least likely to be disturbed, her head tied up to prevent lying down, and orders were given to on no account move her-iodoform pure to be dusted over the part 3 times a day.

On the following morning I set out to see my patient, armed with slings, but to my utter astonishment she was very little worse than on the previous day. I also found she was very "tickle" from the accident, and decided not to attempt to sling her, at all events for the present. She was accordingly simply left tied up, and remained so for a little over 3 weeks, during which time the wound was continually dressed with the iodoform. The opening gradually closed, the discharge - which at no time had been excessive, and which throughout kept its typical character of synovia-ceased, and lameness disappeared. Some slight enlargement of the bones on the 
inner aspect of the hock was left. This received a couple of good blisters of mercuric biniodide, and the mare was able to go to work in exactly 6 wecks, and has remained sound since.

Truly I never saw or heard of an open joint case making such a recovery as this, nor did I ever hear of treatment more simple, though thoroughly aseptic. This rapid recovery was undoubtedly due to the entire absence of inflammation of the structures that enter into the formation of the joint, as evidenced by the astragalus retaining its normal white aspect, and by the synovial membrane not unduly secreting synovia.

No doubt, beyond the hole being directly punched into the capsule, little or no bruising took place; otherwise we should have had inflammation with its complications, and a very different termination of the case.

The plan of treatment laid down by Professor F. Smith does to my mind look feasible and rational after seeing this case, and I shall most certainly make a dependent opening, if such does not exist, in the next case of open joint I get, and, if it demands it, shall also wash it out. It is unquestionably of essential importance that the treatment of open joints should be conducted on strictly aseptic lines.

\section{A CASE OF ASCITES IN THE CAT}

AND

\section{A CASE OF MITRAL INSUFFICIENCY IN THE HORSE.}

By S. Stockman, M.R.C.V.S., Royal Veterinary College, Edinburgh.

CASE I. Ascites.

ON the 22nd September last a tom cat was brought to the Dick College Clinique with the following history.

The animal was aged five years. In June last he had shown considerable enlargement of the abdomen, which disappeared however towards the end of July. About the beginning of September he began to show symptoms of a return of the abdominal enlargement. His appetite fell off, he became languid, disinclined to move, and suffered from thirst. When brought to the college the abdomen was swollen up like a drum and very tense-too tense to allow of one experiencing fluctuation. The buccal mucous membrane was icteric. On examination the heart sounds were found to be normal. The diagnosis was ascites.

Treatment.-I inserted a trochar and canula through the abdominal wall and drew off a pint of albuminous fluid, still leaving a considerable quantity in the cavity. A broad bandage was applied to the abdomen and the following draught prescribed:-

$\mathbf{R}$

Potassii acetatis $z \mathrm{j}$ ss.

Tinct. strophanthi, mm. 40 .

Aquam ad $\overline{3} \mathrm{jj}$.

A dessert spoonful twice a day. 